\title{
Changement climatique et production cotonnière au Sénégal : concevoir autrement les stratégies de diffusion des variétés
}

\author{
Abdoulaye Ndour ${ }^{(1)}$, Romain Loison ${ }^{(2)}$, Jean-Paul Gourlot ${ }^{(2)}$, Karamokho Seydi Ba ${ }^{(1)}$, \\ Abdoulaye Dieng ${ }^{(3)}$, Pascal Clouvel ${ }^{(2)}$ \\ (1) SODEFITEX. Bd du Centenaire de la Commune de Dakar, km 4,5. BP 3216. Dakar (Sénégal). \\ (2) CIRAD. PERSYST. UPR AIDA. Avenue Agropolis. F-34398 Montpellier Cedex 5 (France). \\ E-mail : romain.loison@cirad.fr \\ (3) Université de Thiès. ENSA. BP A 296. Thiès (Sénégal).
}

Reçu le 1 juillet 2016, accepté le 23 janvier 2017.

Cet article est distribué suivant les termes et les conditions de la licence CC-BY (http://creativecommons.org/licenses/by/4.0/ deed.fr)

Description du sujet. L'impact du changement climatique remet en cause la stratégie de diffusion variétale de la Société de Développement et des Fibres Textiles au Sénégal (SODEFITEX), basée sur la distinction de deux variétés : l'une adaptée aux conditions humides et l'autre aux risques de sècheresse. À partir de données recueillies en station expérimentale et en parcelles d'agriculteurs de 2004 à 2011, l'étude évalue la pertinence de cette stratégie.

Objectifs. L'objectif de l'étude est d'évaluer la méthode de sélection variétale et la performance des variétés cultivées et à l'essai, soumises à des environnements hydriques contrastés du fait de la variabilité spatiale et temporelle de la pluviosité et des pratiques culturales.

Méthode. La méthodologie développée permet d'identifier l'occurrence d'un stress hydrique sévère durant les phases critiques de floraison et de développement des capsules vis-à-vis du rendement en coton-graine et la qualité de la fibre. L'indicateur de vécu hydrique produit est ensuite introduit dans l'analyse statistique en tant que co-variable descriptive de l'environnement.

Résultats. L'indicateur produit permet de discriminer les rendements en coton-graine en station expérimentale et chez les producteurs. Bien qu'un effet variétal soit constaté sur le rendement égrenage et les caractéristiques technologiques des fibres, aucune variété ne se distingue des autres vis-à-vis du vécu hydrique.

Conclusions. À défaut de variété adaptée aux stress hydriques, les résultats militent en faveur de l'adoption d'une seule variété sur le bassin de production. La stratégie de diffusion des variétés mériterait toutefois d'être renouvelée pour mieux rendre compte des conditions de culture en milieu réel et permettre l'évaluation des variétés par les producteurs.

Mots-clés. Coton, zone agroclimatique, critère de sélection, variation génétique, stress dû à la sécheresse, expérimentation au champ, Sénégal.

\section{Climate change and cotton production in Senegal: alternate dissemination strategies of varieties}

Description of the subject. Climate change challenges the strategy of varietal diffusion, employed by the Senegalese cotton company (SODEFITEX), based on the distinction of two cultivars: one adapted to humid conditions and the other to drought. Based on data collected in an experimental station and farmer plots from 2004 to 2011, the study provides an assessment of that strategy.

Objectives. The aim of the study was to evaluate both the methodology used for varietal selection and the performance of released and candidate cultivars, under contrasted water environments due to variability in rainfall (spatial and temporal) and crop management.

Method. The methodology used allowed the identification of the occurrence of severe water stress during the critical cotton development phases (flowering and boll development) and its effect on seed cotton yield and fiber quality. The resulting indicator of plant water status was then inserted into our statistical analysis as an environmental covariate.

Results. The resulting indicator allowed the discrimination of seed cotton yield in the experimental station as well as in on-farm experiments. Despite an effect of cultivar on ginning outturn and fiber quality, all cultivars showed similar resilience to water status.

Conclusions. Despite a lack of cultivar adaptation to water stress, results support the adoption of a single cultivar in the whole production basin. However, the diffusion strategy would benefit from a better consideration of farmer cropping conditions, which would thus allow for the evaluation of cultivars by the producers. 
Keywords. Cotton, agroclimatic zones, selection criteria, genetic processes, drought stress, field experimentation, Senegal.

\section{INTRODUCTION}

La pluie est l'une des ressources naturelles les plus importantes pour l'agriculture en l'absence d'irrigation, soit pour la majeure partie de la production agricole en Afrique. La variabilité inter et intra-annuelle des précipitations est donc un des déterminants du succès de l'agriculture dans ces régions (Hulme, 1992). L'étude de l'évolution des conditions climatiques depuis 1960 en Afrique de l'Ouest montre une tendance régionale à l'augmentation significative de la durée des épisodes de sècheresse durant la saison des pluies (Nicholson, 2005 ; New et al., 2006). Au-delà du constat d'une baisse générale de la pluviosité, concomitante à une augmentation de la fréquence des évènements extrêmes au niveau du continent, la complexité des processus climatiques impliqués rend difficile la caractérisation du changement climatique au niveau local en Afrique (Sylla et al., 2013). Au Sénégal, l'analyse des variations climatiques de 1921 à 2014 montre une tendance à la baisse des précipitations annuelles, concomitante à une augmentation de la température moyenne annuelle (Sagna et al., 2015). Sur le plan de la pluviosité, les auteurs distinguent toutefois deux périodes: une première période humide de 1921 à 1967, avec une pluviométrie moyenne de $803 \mathrm{~mm}$ et une deuxième période sèche de 1968 à 2014 avec une moyenne pluviométrique de $614 \mathrm{~mm}$. La période sèche peut se subdiviser en deux sous-périodes, dont la première, de 1968 à 1998, est régulièrement déficitaire à très déficitaire et la deuxième, de 1999 à 2014, est plus variable avec l'apparition de quelques années excédentaires.

Face à ces changements, les sociétés cotonnières sont amenées à adapter leur mode de production et en particulier leur stratégie de choix des variétés à diffuser et de leur aire de culture au sein du bassin de production (carte variétale). En matière de variété, le type de cotonnier (Gossypium hirsutum L.) à croissance indéterminée prédomine en Afrique de l'Ouest. Ce type, caractérisé par une grande vigueur végétative, une floraison abondante et une fructification étalée au cours du cycle de culture confère aux variétés une plasticité remarquable vis-à-vis des aléas climatiques (Sekloka et al., 2008). Toutefois, les pratiques de semis tardif de la part des producteurs exacerbent le problème de la réduction de la durée de la saison des pluies. Au Cameroun, face à ce problème, la sélection de variétés précoces, voire de types variétaux plus déterminés, a donné des résultats prometteurs sur le plan de la productivité et de la qualité des fibres produites (Cao et al., 2011).
Avec $46 \%$ du territoire national et $27 \%$ de la population totale du Sénégal, le bassin de production cotonnière recouvre les régions naturelles du Sénégal oriental, de la Casamance continentale et le Sud du Sine-Saloum. Conformément au gradient de pluviosité décrit par Ndong (1995) et Dacosta et al. (2002), la Société de Développement et des Fibres Textiles du Sénégal (SODEFITEX) distingue trois zones agroclimatiques :

- une zone sèche au Nord-Ouest du bassin cotonnier, avec une pluviosité moyenne variant entre 600 et $800 \mathrm{~mm}$,

- une zone humide à l'Est et à l'Ouest du Parc national du Niokolo-Koba avec une pluviosité supérieure à $900 \mathrm{~mm}$ et pouvant dépasser $1200 \mathrm{~mm}$,

- une zone intermédiaire située dans la partie nord-est du bassin et sud de la frontière sud avec la Gambie, avec une pluviosité comprise entre 800 et $900 \mathrm{~mm}$.

La contribution des zones à la production de coton-graine se situe respectivement à hauteur de 60 , 30 et $10 \%$ pour les zones agro-climatiques humides, intermédiaires et sèches, pour une production totale variant de 25000 à $50000 \mathrm{t}$ de coton-graine, selon les années. Sur le plan technique, ce zonage agro-climatique s'accompagne de recommandations distinctes en matière de fertilisation minérale notamment. Si la SODEFITEX recommande des pratiques de fertilisation différentes selon la zone agro-climatique, c'est afin de limiter le risque économique lié à l'occurrence de conditions hydriques défavorables susceptibles de limiter l'efficience d'utilisation des engrais vis-à-vis du rendement en coton-graine. En ce qui concerne la carte variétale toutefois, et bien que les variétés adaptées aux conditions humides et aux stress hydriques se retrouvent respectivement au Sud et au Nord du bassin de production, leur aire de culture respective ne correspond pas aux délimitations des zones agro-climatiques et varie au cours du temps. Cette distinction spatiale de trois zones agro-climatiques différenciées et de la carte variétale à deux aires de cultures est aujourd'hui remise en question par l'évolution récente de la pluviosité au sein du bassin de production. L'importante variabilité annuelle et spatiale de pluviosité enregistrée ces dernières années atténue les contrastes entre zones agro-climatiques. Outre l'occurrence de stress hydrique lors des «pauses pluviométriques » au cours de la saison des pluies (Salack et al., 2012), l'incertitude sur l'installation des pluies en début d'hivernage entraine un retard général des semis (Soumaré et al., 2008) avec pour conséquence un risque accru de déficit hydrique en fin de cycle de culture. 
Face à ce phénomène, l'objectif de l'étude est d'évaluer la performance des variétés de cotonnier cultivées et à l'essai au Sénégal, et soumises à une forte variabilité interannuelle de pluviosité en lien avec le changement climatique local décrit par Sagna et al. (2015). L'article décrit la démarche proposée pour caractériser de façon formelle l'environnement hydrique des plantes et estimer le risque de stress hydrique en cours de cycle par voie de modélisation informatique selon une méthode inspirée des travaux de Loison (2015).

\section{MATÉRIEL ET MÉTHODES}

\subsection{Le dispositif d'étude}

Le dispositif d'étude utilise les données recueillies par la SODEFITEX de 2004 à 2011 sur ses sites de recherche en milieu contrôlé au niveau d'Antennes Multilocales d'Expérimentation (AMEX) et chez les producteurs dans les Sites de Recherche et Développement (SRD). Ces dispositifs permettent d'évaluer l'intérêt de variétés candidates au renouvellement des variétés en cours de culture, en comparaison avec les témoins que sont les variétés déjà diffusées sur la même aire de culture.
Les sites expérimentaux. Le réseau d'AMEX comporte cinq antennes : Syllacounda et Dabo pour la zone agro-climatique humide, Vélingara et Dialacoto pour la zone intermédiaire, et Koussanar pour la zone sèche (Figure 1). Les dispositifs expérimentaux sont en blocs randomisés, avec 4 ou 5 répétitions selon les sites et années. Le réseau de SRD comporte cinq sites : Ibel et Sare Hamidou pour la zone agro-climatique humide, Medina Baoussou et Sare Bourang pour la zone intermédiaire, et Aïnoumane pour la zone sèche. Dans chaque SRD, les expérimentations sont réparties chez quatre producteurs différents, à raison d'un bloc par producteur, implanté au sein d'un champ cultivé pour la production cotonnière. Les travaux culturaux sont réalisés par les producteurs de façon identique à celle du champ cultivé dans lequel le bloc expérimental est implanté. En termes d'environnement, un tel dispositif sans répétition induit une diversité de conditions de milieu et de pratiques d'un bloc paysan à l'autre au sein d'un même SRD. Par contre, ce dispositif est conçu pour estimer la capacité d'adaptation du nouveau matériel végétal aux pratiques réelles des producteurs, en dehors des stations de recherche.

En condition d'AMEX comme de SRD, chaque objet occupe une parcelle élémentaire de 6 lignes de $10 \mathrm{~m}$. Les lignes de semis sont espacées de $80 \mathrm{~cm}$ et les poquets de $30 \mathrm{~cm}$ dans la ligne, à raison de 3 à

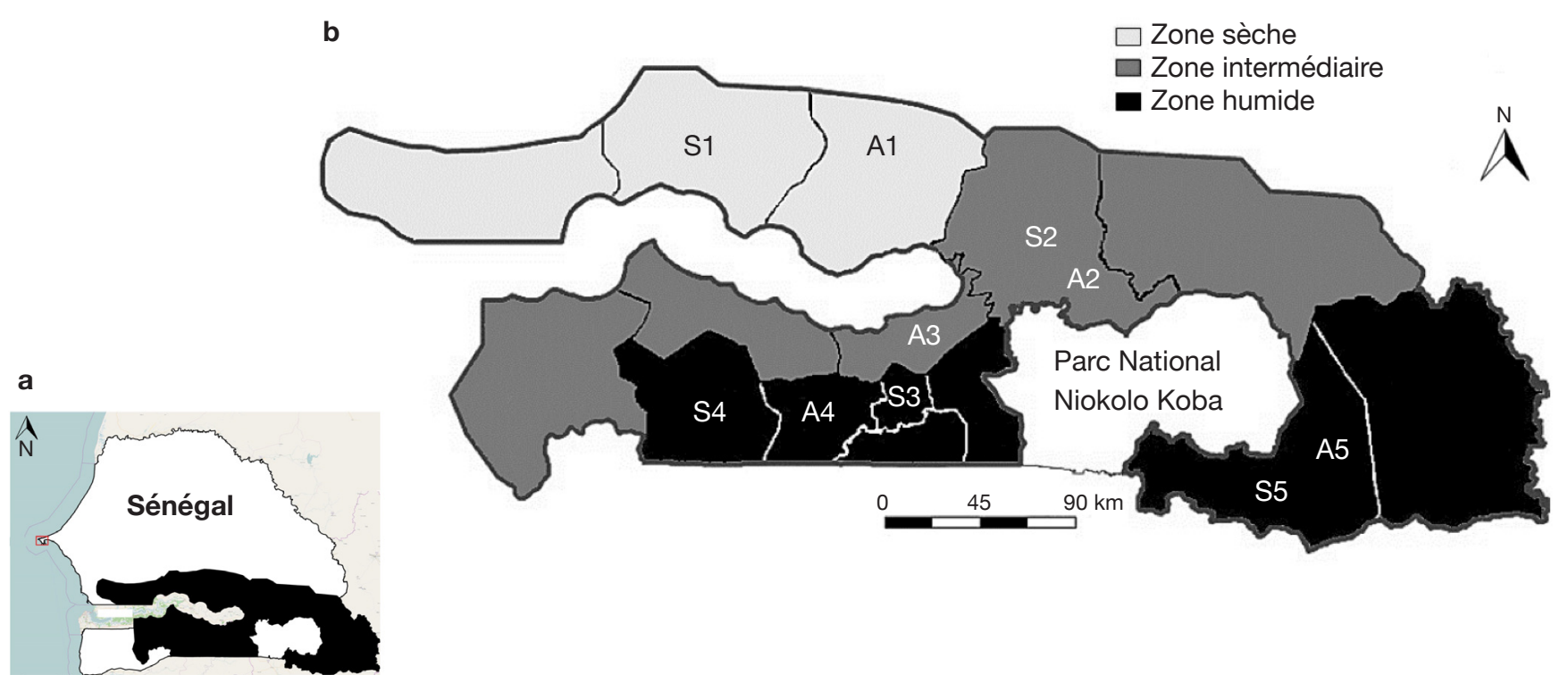

Figure 1. Bassin de production cotonnière au Sénégal (a) et zonage agro-climatique du bassin de production cotonnière de la SODEFITEX (b) - Cotton production area in Senegal (a) and agro-climatic zones of the SODEFITEX cotton production $\operatorname{area}(\boldsymbol{b})$.

Zone sèche en gris clair, intermédiaire en gris foncé et humide en noir. Le dispositif de recherche comporte 5 Antennes Multi locales d'Expérimentation ou AMEX : Koussanar (A1), Dialacoto (A2), Vélingara (A3), Dabo (A4) et Syllacounda (A5); et 5 Sites de Recherche et Développement ou SRD : Aïnoumane (S1), Medina Baoussou (S2), Sare Bourang (S3), Sare Hamidou (S4), et Ibel (S5) - Dry zone in light grey, intermediate zone in dark grey, and wet zone in black. The research device includes 5 experimental stations or AMEX: Koussanar (A1), Dialacoto (A2), Vélingara (A3), Dabo (A4) and Syllacounda (A5); and 5 Sites for Research and Development or SRD: Ä̈noumane (S1), Medina Baoussou (S2), Sare Bourang (S3), Sare Hamidou (S4), and Ibel (S5). 
4 plantes par poquet en semis manuel en AMEX et 6 à 7 plantes par mètre linéaire en semis mécanique au semoir à traction animale en SRD (disque 16 trous). Après démariage manuel, les densités enregistrées varient respectivement de $6,9 \pm 1,8$ en condition d'AMEX et de $5,1 \pm 1,7$ plantes $\cdot \mathrm{m}^{-2}$ en SRD. $\grave{A}$ titre d'information, la densité recommandée par la SODEFITEX après démariage est de 8 plants $\cdot \mathrm{m}^{-2}$. Les dates de levée s'étalent du 21/6 (Syllacounda 2005) au 20/7 (Dialacoto 2009) en condition d'AMEX et du 21/6 (Ibel 2005) au 29/7 (Aïnoumane 2007) en condition de SRD.

En termes de pluviosité enfin, et à l'appui de l'argumentaire sur le changement climatique au niveau du bassin de production, on observe une forte variabilité interannuelle avec l'occurrence d'années humides en 2004, 2010 et d'années déficitaires en 2006 et 2011 (Figure 2). De plus, alors que les écarts de pluviosité entre zones agro-climatiques humide et sèche restent relativement stables d'une année à l'autre, marquant ainsi le contraste entre les deux zones, ce n'est pas le cas de la zone intermédiaire dont la pluviosité s'approche de celle de la zone sèche certaines années et de la zone humide d'autres années.

Les tests variétaux. Entre 2004 et 2011, trois variétés étaient diffusées : la variété Stam 42 jusqu'en 2006 puis Stam 279A à partir de 2007 comme variétés adaptées aux conditions de culture humides sur la carte variétale et Isco PG comme variété adaptée aux conditions plus sèches sur cette même carte (Figure 3). La limite entre les deux aires de culture que ces variétés occupent varie dans le temps. En référence au catalogue CORAF/ WECARD (2005), la variété Isco PG (obtention de l'Institut Sénégalais de Recherche Agricole) est relativement adaptée à la sècheresse et présente un cycle plus court que Stam 42, mais de même durée que celui de Stam 279A, toutes deux obtentions de l'ITRA (Institut Togolais de Recherche Agronomique).
Trois variétés candidates ont été testées durant cette période: Stam 59A et Stam 129A (obtentions ITRA), ainsi que FK 59K (obtention de l'Institut de l'Environnement et Recherches Agricoles du Burkina Faso). Les tests conduits de 2004 à 2011 en AMEX et SRD sont présentés dans les tableaux 1 et $\mathbf{2}$. D'un point de vue génétique, Stam 59A, Stam 129A et Stam 279A ont été obtenues par re-sélection de Stam F,

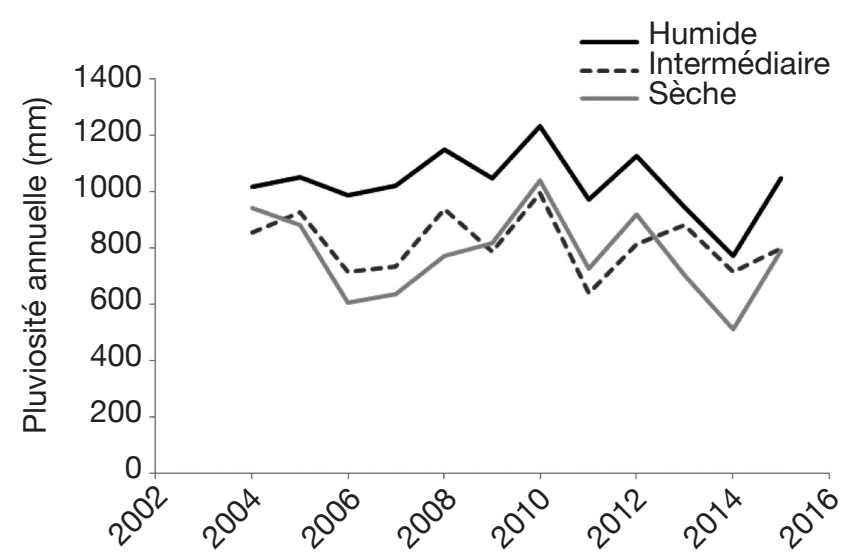

Figure 2. Pluviosité annuelle moyenne enregistrée sur le bassin de production cotonnière du Sénégal, par zone agroclimatique entre 2004 et 2015 - Average annual rainfall recorded on the Senegalese cotton production area by agroclimate zone from 2004 to 2015.

Zone humide (ligne noire), zone intermédiaire (pointillés) et zone sèche (gris léger). Les relevés pluviométriques proviennent des stations météorologiques de Dabo, Kounkane, Kolda et Kedougou pour la zone humide, de Tambacounda, Missirah, Dialacoto et Vélingara pour la zone intermédiaire, et de Nioro, Koussanar et Koungheul pour la zone sèche - Wet zone (black line), Intermediate zone (dotted) and Dry zone (light grey). Rainfall data were collected in the meteorological stations of Dabo, Kounkane, Kolda and Kedougou for the wet zone, Tambacounda Missirah Dialacoto and Vélingara for the intermediate zone, and Nioro, Koussanar and Koungheul for the dry zone.
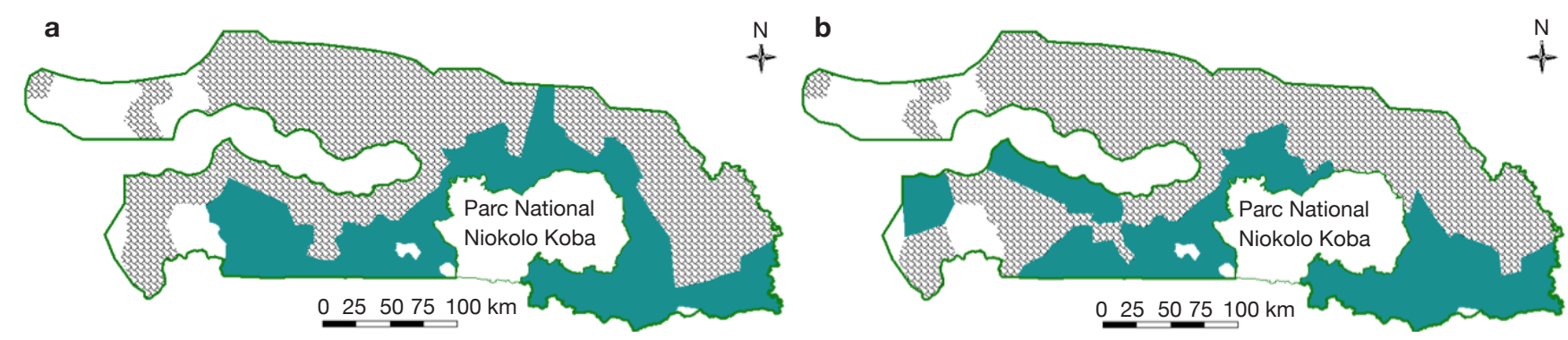

Figure 3. Répartition spatiale (carte variétale) des variétés cultivées dans le bassin de production cotonnière sénégalais en 2004 (a) et à partir de 2011 (b) - Spatial distribution of cotton varieties (distribution map) in Senegal in 2004 (a) and 2011 (b).

Isco PG adaptée aux conditions plus sèches (pointillé) et Stam 42 (a) puis Stam 279A (b) aux conditions humides (uni) - Isco PSG for drought-prone conditions (dotted) and Stam 42 (a) Stam 279A (b) for wet conditions (plain). 
Tableau 1. Tests variétaux réalisés en antennes multilocales d'expérimentation (AMEX) - Varietal tests in experimental stations (AMEX).

\begin{tabular}{|c|c|c|c|c|c|}
\hline \multirow[t]{2}{*}{ Campagne } & \multirow{2}{*}{$\begin{array}{l}\text { Zone sèche } \\
\text { Koussanar }\end{array}$} & \multicolumn{2}{|c|}{ Zone intermédiaire } & \multicolumn{2}{|l|}{ Zone humide } \\
\hline & & Vélingara & Dialacoto & Dabo & Syllacounda \\
\hline 2004 & $\begin{array}{l}\text { Stam 42 } \\
\text { Stam 279A }\end{array}$ & $\begin{array}{l}\text { Stam 42 } \\
\text { Stam 279A }\end{array}$ & $\begin{array}{l}\text { Stam } 42 * \\
\text { Stam 279A }\end{array}$ & $\begin{array}{l}\text { Stam 42* } \\
\text { Stam 279A }\end{array}$ & $\begin{array}{l}\text { Stam 42* } \\
\text { Stam 279A }\end{array}$ \\
\hline 2005 & $\begin{array}{l}\text { Isco PG* } \\
\text { Stam 279A } \\
\text { Stam 59A } \\
\text { FK 59K }\end{array}$ & $\begin{array}{l}\text { Isco PG* } \\
\text { Stam 279A } \\
\text { Stam 59A } \\
\text { FK 59K }\end{array}$ & $\begin{array}{l}\text { Stam } 42 * \\
\text { Stam 279A } \\
\text { Stam 59A } \\
\text { FK 59K }\end{array}$ & $\begin{array}{l}\text { Stam } 42^{*} \\
\text { Stam 279A } \\
\text { Stam 59A } \\
\text { FK 59K }\end{array}$ & $\begin{array}{l}\text { Stam } 42^{*} \\
\text { Stam 279A } \\
\text { Stam 59A } \\
\text { FK 59K }\end{array}$ \\
\hline 2006 & $\begin{array}{l}\text { Isco PG* } \\
\text { Stam 59A } \\
\text { FK 59K }\end{array}$ & $\begin{array}{l}\text { Isco PG* } \\
\text { Stam 59A } \\
\text { FK 59K }\end{array}$ & $\begin{array}{l}\text { Stam } 42 * \\
\text { Stam 59A } \\
\text { FK 59K }\end{array}$ & $\begin{array}{l}\text { Stam } 42 * \\
\text { Stam 59A } \\
\text { FK 59K }\end{array}$ & $\begin{array}{l}\text { Stam } 42 * \\
\text { Stam 59A } \\
\text { FK 59K }\end{array}$ \\
\hline 2009 & $\begin{array}{l}\text { Isco PG* } \\
\text { Stam 129A }\end{array}$ & $\begin{array}{l}\text { Isco } \mathrm{PG}^{*} \\
\text { Stam 129A }\end{array}$ & $\begin{array}{l}\text { Isco PG* } \\
\text { Stam 129A }\end{array}$ & $\begin{array}{l}\text { Stam 279A* } \\
\text { Stam 129A }\end{array}$ & $\begin{array}{l}\text { Stam 279A* } \\
\text { Stam 129A }\end{array}$ \\
\hline 2010 & $\begin{array}{l}\text { Isco PG* } \\
\text { Stam 129A } \\
\text { Stam 279A }\end{array}$ & $\begin{array}{l}\text { Isco PG* } \\
\text { Stam 129A } \\
\text { Stam 279A }\end{array}$ & $\begin{array}{l}\text { Isco PG* } \\
\text { Stam 129A } \\
\text { Stam 279A }\end{array}$ & $\begin{array}{l}\text { Isco PG } \\
\text { Stam 129A } \\
\text { Stam 279A* }\end{array}$ & $\begin{array}{l}\text { Isco PG } \\
\text { Stam 129A } \\
\text { Stam 279A* }\end{array}$ \\
\hline 2011 & $\begin{array}{l}\text { Isco PG* } \\
\text { Stam 129A } \\
\text { Stam 279A }\end{array}$ & $\begin{array}{l}\text { Isco PG* } \\
\text { Stam 129A } \\
\text { Stam 279A }\end{array}$ & $\begin{array}{l}\text { Isco PG* } \\
\text { Stam 129A } \\
\text { Stam 279A }\end{array}$ & $\begin{array}{l}\text { Isco PG } \\
\text { Stam 129A } \\
\text { Stam 279A* }\end{array}$ & $\begin{array}{l}\text { Isco PG } \\
\text { Stam 129A } \\
\text { Stam 279A* }\end{array}$ \\
\hline
\end{tabular}

Les variétés dont le nom est suivi d'un astérisque correspondent aux variétés témoins utilisées localement en production - Cultivars whose names are followed by an asterisk are the control cultivars used locally in production.

Tableau 2. Tests variétaux réalisés en sites de recherche et développement (SRD) - Varietal tests in farmer field conditions (SRD).

\begin{tabular}{|c|c|c|c|c|c|}
\hline \multirow[t]{2}{*}{ Campagne } & \multirow{2}{*}{$\frac{\text { Zone sèche }}{\text { Aïnoumane }}$} & \multicolumn{2}{|c|}{ Zone intermédiaire } & \multicolumn{2}{|l|}{ Zone humide } \\
\hline & & M. Baoussou & S. Hamidou & S. Bourang & Ibel \\
\hline 2005 & $\begin{array}{l}\text { Isco PG* } \\
\text { Stam 279A }\end{array}$ & $\begin{array}{l}\text { Stam 42* } \\
\text { Stam 279A }\end{array}$ & $\begin{array}{l}\text { Stam } 42 * \\
\text { Stam 279A }\end{array}$ & $\begin{array}{l}\text { Stam } 42 * \\
\text { Stam 279A }\end{array}$ & $\begin{array}{l}\text { Stam } 42 * \\
\text { Stam 279A }\end{array}$ \\
\hline 2007 & $\begin{array}{l}\text { Isco PG* } \\
\text { Stam 59A } \\
\text { FK 59K }\end{array}$ & $\begin{array}{l}\text { Isco PG* } \\
\text { Stam 59A } \\
\text { FK 59K }\end{array}$ & $\begin{array}{l}\text { Stam 279A* } \\
\text { Stam 59A } \\
\text { FK 59K }\end{array}$ & $\begin{array}{l}\text { Stam 279A* } \\
\text { Stam 59A } \\
\text { FK 59K }\end{array}$ & $\begin{array}{l}\text { Stam 279A* } \\
\text { Stam 59A } \\
\text { FK 59K }\end{array}$ \\
\hline 2008 & $\begin{array}{l}\text { Isco PG* } \\
\text { Stam 59A } \\
\text { FK 59K }\end{array}$ & $\begin{array}{l}\text { Isco PG* } \\
\text { Stam 59A } \\
\text { FK 59K }\end{array}$ & $\begin{array}{l}\text { Isco PG* } \\
\text { Stam 59A } \\
\text { FK 59K }\end{array}$ & $\begin{array}{l}\text { Stam 279A* } \\
\text { Stam 59A } \\
\text { FK 59K }\end{array}$ & $\begin{array}{l}\text { Stam 279A* } \\
\text { Stam 59A } \\
\text { FK 59K }\end{array}$ \\
\hline 2011 & & $\begin{array}{l}\text { Isco PG* } \\
\text { Stam 129A } \\
\text { Stam 279A }\end{array}$ & $\begin{array}{l}\text { Isco PG* } \\
\text { Stam 129A } \\
\text { Stam 279A }\end{array}$ & $\begin{array}{l}\text { Isco PG } \\
\text { Stam 129A } \\
\text { Stam 279A* }\end{array}$ & $\begin{array}{l}\text { Isco PG } \\
\text { Stam 129A } \\
\text { Stam 279A* }\end{array}$ \\
\hline
\end{tabular}

Les variétés dont le nom est suivi d'un astérisque correspondent aux variétés témoins utilisées localement en production - Cultivars whose names are followed by an asterisk are the control cultivars used locally in production.

variété largement cultivée en Afrique de l'Ouest et du Centre depuis 25 ans. La variété FK 59K a été obtenue par croisement entre Stam 59A et F135 (un des parents de Stam F). Ces variétés présentent donc une faible distance génétique entre elles et s'apparentent toutes au type de cotonnier à croissance indéterminée.

Les données recueillies. La pluviosité est enregistrée par une station météorologique sur chaque site expérimental (5 AMEX et 5 SRD). Les observations agronomiques sont réalisées sur les 4 lignes centrales de chaque parcelle élémentaire par le personnel technique de la SODEFITEX. La date de levée est notée lorsqu'au moins $50 \%$ des poquets présentent des plantules au stade cotylédons apparents. Les dates d'apparition de la $1^{\text {ère }}$ fleur, de fin de la floraison utile, d'ouverture de la $11^{\text {ère }}$ capsule (déhiscence du cotongraine) ont été recueillies sur 10 plantes par parcelle 
élémentaire. La date retenue pour chaque stade correspond à l'occurrence de l'évènement sur $50 \%$ des plantes. Le nombre de nœuds de la tige principale au-dessus du rameau portant une fleur blanche sur son premier nœud (NAWF) diminue durant la phase de floraison. La fin de la floraison utile est estimée lorsque cet indicateur prend une valeur de 3 . Les fleurs apparues au-delà de cette date ne contribuent pas à la production (Oosterhuis et al., 1993). Le rendement en coton-graine est obtenu par récoltes successives de la totalité des capsules arrivées à déhiscence et pesées après séchage. Le rendement à l'égrenage $(\% \mathrm{~F})$ correspond au pourcentage de fibre obtenu après égrenage (égreneuse 10 scies, Continental Gin Company, Huller Cleaner Feader, Model E-PF) par rapport à la masse initiale de coton-graine de l'échantillon. Le rendement en fibre par hectare est obtenu en multipliant le rendement en coton-graine par le rendement à l'égrenage.

En ce qui concerne les caractéristiques commerciales enfin, la qualité des fibres a été mesurée sur le coton produit dans les cinq sites AMEX en 2006 et 2009, à raison d'une caractérisation d'un échantillon moyen représentatif par variété et par parcelle élémentaire. Les caractéristiques de longueur, ténacité, maturité et couleur des fibres déterminent le classement des lots de coton produits (Cotton Incorporated, 2013) et donc le marché sur lequel ils pourront être commercialisés et leur prix. L'analyse a été réalisée au Laboratoire de Technologie et de Caractérisation des fibres naturelles du CIRAD à Montpellier, accrédité ISO
$17025\left(\mathrm{~N}^{\circ}\right.$ COFRAC 1-2386). Les caractéristiques technologiques des fibres (micronaire, longueur, uniformité, ténacité et couleur) ont été mesurées sur chaine de mesure instrumentale SITC (Standardized Instrument for Testing Cotton) Uster Technologies ${ }^{\circledR}$ HVI $1000^{\mathrm{TM}}$. Les caractéristiques de maturité et de finesse de fibre ont été mesurées sur l'appareil Fineness Maturity Tester de Shriley Development limited.

\subsection{Traitement des données}

Cadre d'analyse. Compte-tenu de la variabilité interannuelle de pluviosité observée au niveau du bassin de production (Figure 2) et de l'étalement des dates de levée des plantes sur les différents sites expérimentaux (Tableau 3), le regroupement des tests par zone agro-climatique n'est pas pertinent pour accéder à l'interaction entre variété et environnement hydrique. Pour contourner la difficulté, nous proposons d'introduire le vécu hydrique estimé des cotonniers dans l'analyse statistique en tant que co-variable descriptive de l'environnement hydrique et en complément d'aspects locaux indissociables du site d'étude (sol, itinéraire technique).

Estimation du vécu hydrique des cotonniers. Afin d'estimer cette variable, nous avons d'abord réalisé une typologie d'environnement hydrique des cotonniers en croisant typologie de pluviosité enregistrée localement et période de levée des cotonniers pour

Tableau 3. Variabilité d'environnement hydrique des cotonniers explorée dans le dispositif et prise en compte dans les simulations - Variability of water environment of cotton plants explored in the device and taken into account in the simulations.

\begin{tabular}{|c|c|c|c|c|c|}
\hline \multirow[t]{2}{*}{ Levée } & \multirow[t]{2}{*}{ Pluviosité } & \multicolumn{2}{|l|}{ AMEX } & \multicolumn{2}{|l|}{ SRD } \\
\hline & & Dispositif & Simulation & Dispositif & Simulation \\
\hline L1 : 1 au 30 juin & $\begin{array}{l}\text { A } \\
\text { B } \\
\text { C } \\
\text { D }\end{array}$ & $\begin{array}{l}1 \\
2 \\
1\end{array}$ & $\begin{array}{l}1 \\
1\end{array}$ & $\begin{array}{l}4 \\
1 \\
2\end{array}$ & 1 \\
\hline L2 : 1 au 15 juin & $\begin{array}{l}\text { A } \\
\text { B } \\
\text { C } \\
\text { D }\end{array}$ & $\begin{array}{l}4 \\
7 \\
7 \\
5\end{array}$ & $\begin{array}{l}1 \\
4 \\
3 \\
2\end{array}$ & $\begin{array}{l}20 \\
26 \\
5\end{array}$ & $\begin{array}{l}2 \\
3 \\
2\end{array}$ \\
\hline L3 : 16 au 25 juillet & $\begin{array}{l}\text { B } \\
\text { C } \\
\text { D }\end{array}$ & $\begin{array}{l}1 \\
2\end{array}$ & $\begin{array}{l}1 \\
2\end{array}$ & $\begin{array}{l}2 \\
6 \\
1\end{array}$ & $\begin{array}{l}1 \\
2 \\
1\end{array}$ \\
\hline L4 : après le 25 juillet & $\mathrm{D}$ & & & 4 & 1 \\
\hline Effectif total & & 30 & 15 & 71 & 13 \\
\hline
\end{tabular}

A : installation des pluies précoces (début mai), pluviosité annuelle $>1000 \mathrm{~mm}$ et régulière - early arrival of rains (early May), annual rainfall $>1,000 \mathrm{~mm} ; \mathbf{B}$ : installation retardée (fin mai - début juin) et pluviosité annuelle $>1000$ mm et régulière - late arrival of rains (late May-early June) and annual rainfall $>1,000 \mathrm{~mm}$ and steady; $\mathbf{C}$ : installation retardée et pluviosité annuelle comprise entre 800 et $1000 \mathrm{~mm}$ - late arrival of rains and annual rainfall between 800 and 1,000 $\mathrm{mm}$; D : installation retardée et pluviosité annuelle $<800 \mathrm{~mm}-$ late arrival of rains and annual rainfall $<800 \mathrm{~mm}$. 
chacun des tests variétaux. Ensuite, nous avons réalisé une simulation du vécu hydrique des cotonniers pour 28 situations expérimentales représentatives de la diversité d'environnement hydrique explorée dans l'étude (Tableau 3) par voie de modélisation à l'aide du logiciel DSSAT CSM-CROPGRO-Cotton (Jones et al., 2003). Enfin, nous avons établi une règle de correspondance entre environnement hydrique et vécu hydrique des cotonniers, puis appliqué cette règle aux 101 cas de l'étude (Tableau 3).

Typologie de pluviosité. À partir des pluviosités enregistrées dans les différents sites expérimentaux et sur la base des critères retenus par l'encadrement de la SODEFITEX pour le suivi des campagnes agricoles, nous avons distingué quatre types de pluviosité selon la précocité d'installation de la saison des pluies et l'abondance et la régularité des pluies durant l'hivernage (Figure 4) :

- Type A : installation des pluies précoce (début mai), pluviosité $>1000 \mathrm{~mm}$ et régulière ;

- Type B : installation retardée (fin mai-début juin), pluviosité $>1000 \mathrm{~mm}$ et régulière ;

- Type C: installation retardée, pluviosité annuelle comprise entre 800 et $1000 \mathrm{~mm}$;

- Type D: installation retardée, pluviosité annuelle $<800 \mathrm{~mm}$.

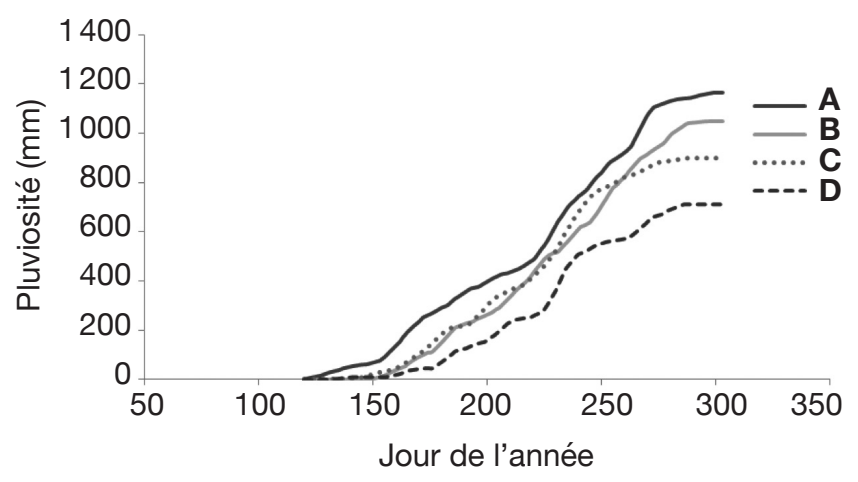

Figure 4. Typologie de pluviosité enregistrée sur le bassin de production cotonnière au Sénégal de 2004 à 2011 - Typology of rainfall recorded in the cotton production area in Senegal from 2004 to 2011.

A: Kédougou 2005 : installation des pluies précoce (début mai), pluviosité annuelle $>1000 \mathrm{~mm}$ et régulière - early arrival of rains (early May), annual rainfall $>1000 \mathrm{~mm}$ and regular; B : Vélingara 2006 : installation retardée (fin mai-début juin), pluviosité annuelle $>1000 \mathrm{~mm}$ et régulière - late arrival of rains (late May-early June), annual rainfall $>1,000 \mathrm{~mm}$ and regular; C : Missira 2008 : installation retardée, pluviosité annuelle comprise entre 800 et $1000 \mathrm{~mm}$ - late arrival of rains, annual rainfall between 800 and $1000 \mathrm{~mm} ; \mathbf{D}$ : Missira 2007 : installation retardée, pluviosité annuelle $<800 \mathrm{~mm}$ - late arrival of rains, annual rainfall $<800 \mathrm{~mm}$.
Sur les 50 cas de pluviosité explorés dans l'étude (AMEX : 5 lieux * 6 années + SRD : 5 lieux * 4 années), on compte $12 \%$ de type A, $30 \%$ de type B, $38 \%$ de type $\mathrm{C}$ et $20 \%$ de type $\mathrm{D}$. Les types de pluviosité $\mathrm{A}, \mathrm{B}, \mathrm{C}$ et $\mathrm{D}$ se répartissent respectivement à hauteur de $25,38,38$ et $0 \%$ pour la zone agroclimatique humide, de $0,25,31$ et $44 \%$ pour la zone intermédiaire et de $10,10,30$ et $50 \%$ pour la zone sèche.

Environnement hydrique des cotonniers. Cet environnement est obtenu par le croisement des typologies de pluviosité avec la période de levée de la culture. En cohérence avec les recommandations techniques de la SODEFITEX auprès des producteurs, 4 plages de levée ont été identifiées :

- L1 (hautement recommandée) : levée du $1^{\text {er }}$ au 30 juin ;

- L2 (recommandée) : levée du $1^{\text {er }}$ au 15 juillet ;

- L3 (tolérée) : levée du 16 au 25 juillet;

- L4 (exclue) : levée après le 25 juillet.

La diversité d'environnement hydrique explorée dans l'étude est montrée dans le tableau 3. Quel que soit le type de pluviosité enregistré, L2 est la plage de levée la plus fréquente avec $73 \%$ des cas. Les levées tardives (L3 et L4) représentent $16 \%$ des cas et les levées précoces (L1) $11 \%$ des cas.

Simulation du vécu hydrique des cotonniers. Selon une méthodologie décrite par Loison (2015), nous avons procédé à une simulation du vécu hydrique des cotonniers sur un pas de temps quotidien à l'aide du logiciel DSSAT (Jones et al., 2003). Les simulations ont été faites sur un échantillon représentatif (28 simulations pour les 101 cas observés) de la diversité d'environnement hydrique explorée dans le dispositif (Tableau 3). Les paramètres variétaux adoptés pour la modélisation (phénologie) ont été ceux estimés pour la variété témoin Stam 279A. En termes de variable d'entrée, nous avons opté pour un sol moyen correspondant à une texture sablolimoneuse et une profondeur de $100 \mathrm{~cm}$. Outre la pluviosité, mesurée sur chaque site expérimental, les variables climatiques de rayonnement incident et la température journalière proviennent de la NASA (2016). Concernant les opérations culturales, nous avons considéré une densité unique de 7 plantes $\cdot \mathrm{m}^{-2}$. La fertilisation minérale appliquée a été de $200 \mathrm{~kg} \cdot \mathrm{ha}^{-1}$ d'engrais complet NPKSB $(22 \%-10 \%-15 \%-5 \%-1 \%)$, soit $44 \mathrm{~kg} \mathrm{~N} \cdot \mathrm{ha}^{-1}$ à la levée, complétée par $50 \mathrm{~kg} \cdot \mathrm{ha}^{-1}$ d'urée $(46 \%)$ soit $23 \mathrm{~kg} \mathrm{~N} \cdot \mathrm{ha}^{-1}$ à la floraison pour les zones agroclimatiques humides et intermédiaires et de $150 \mathrm{~kg}$.

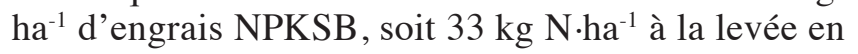
zone sèche, sans complément d'urée. 
La date de levée considérée est celle observée sur chaque site expérimental et chaque année. La variable d'état hydrique simulée sur un pas de temps journalier par DSSAT varie de 0 à 1 . Une valeur comprise entre 0 et 0,4 correspond à un stress hydrique modéré, susceptible d'induire un ralentissement de la croissance des cotonniers sans altérer le développement des fruits déjà formés. Une valeur de 0,6 et au-delà correspond à un stress sévère, susceptible d'altérer la photosynthèse et par voie de conséquence l'accumulation de biomasse par la plante et d'entrainer l'abscission des boutons floraux, des fleurs et des jeunes capsules en formation (Sadras \& Milroy, 1996).

La qualité de la calibration du modèle a été évaluée sur la base des rendements en coton-graine obtenus et simulés en condition expérimentale d'AMEX. Les résultatsobtenus $\left(\mathrm{R}^{2}>0,9\right.$, Figure 5) suggèrentuneprise en compte satisfaisante de l'environnement hydrique des cotonniers par le modèle en condition d'AMEX. Réalisées sans modifier le paramétrage du modèle, les simulations obtenues en condition de SRD surestiment par contre sensiblement le rendement en coton-graine observé. Les données recueillies ne permettent pas d'en identifier la cause, vraisemblablement liée à une conduite sub-optimale de la culture spécifique de chaque agriculteur pour la densité de plantes par hectare, et le contrôle des mauvaises herbes et des ravageurs. Dans cette hypothèse, la présence de mauvaises herbes comme la présence de ravageurs contribuent à une baisse du rendement en coton-graine

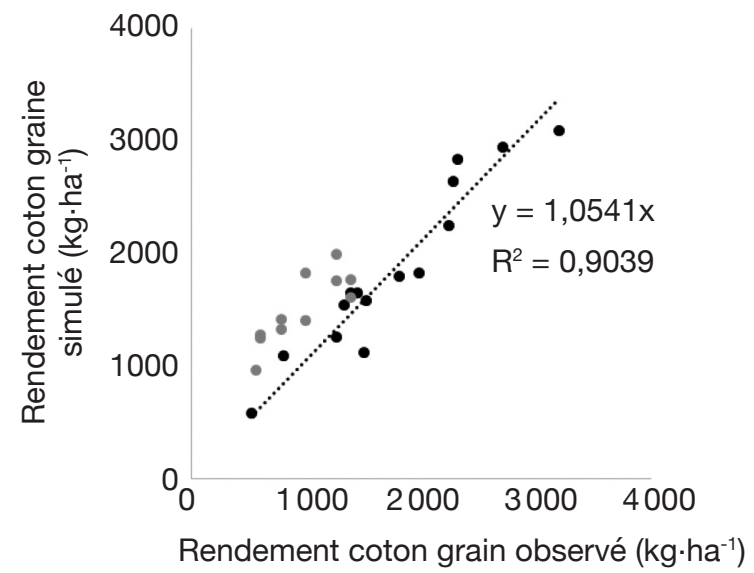

Figure 5. Validation de la simulation du rendement en cotongraine à l'aide du logiciel DSSAT (Jones et al., 2003) en condition de station expérimentale AMEX (points noirs). La simulation des situations réelles en condition de SRD à partir du même paramétrage du modèle conduit à une surestimation sensible du rendement observé (points gris) - Validation of seed cotton yield simulation using the DSSAT software (Jones et al., 2003), in conditions of experimental station AMEX (black points). Simulation of farmers' fields seed cotton yield using the same set of parameters led to an overestimation of the observed yield (grey points).
(Badiane et al., 2015). Du fait de la compétition pour l'eau, les nutriments et la lumière, les mauvaises herbes limitent le développement végétatif des plantes cultivées mais contribuent toutefois à la transpiration globale du couvert végétal (Lopes et al., 2004). Par ailleurs, les ravageurs, carpophages pour l'essentiel, contribuent à une baisse du rendement en coton-graine sans altération du développement végétatif des plantes et donc de la transpiration. À défaut de précision sur l'état des cultures, nous admettrons que le bilan hydrique simulé rend compte du vécu hydrique des cotonniers en condition de SRD.

Estimation du vécu hydrique des cotonniers. Les 28 simulations réalisées (Figure 6) ont permis de distinguer les environnements n'ayant pas entrainé de stress en cours de culture (SH0) des environnements ayant entrainé un stress hydrique durant le cycle de culture (SH1). En cohérence avec la littérature (Sadras \& Milroy, 1996), l'occurrence d'un stress hydrique a été considérée comme effective pour des niveaux de stress supérieur ou égal à 0,6 avant ouverture de la première capsule. Au-delà de ce stade phénologique, le stress hydrique n'a pas d'effet négatif sur le rendement et peut améliorer la qualité de la production (de Kock et al., 1990).

Pour un environnement hydrique donné, quand toutes les simulations donnent le même vécu hydrique, alors c'est celui-là qui est retenu, $\mathrm{SH} 0$ ou $\mathrm{SH} 1$ selon les cas. Quand les simulations donnent des vécus hydriques différents, ce qui est retenu est l'occurrence d'un stress en cours de cycle (SH1). Le tableau 4 donne la correspondance entre environnement hydrique et

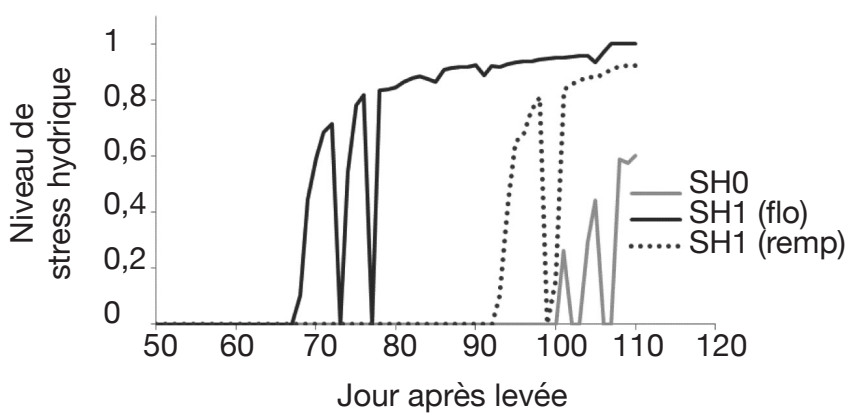

Figure 6. Exemples de vécu hydrique des cotonniers simulé par DSSAT - Examples of water stress experienced by cotton plants using the DSSAT crop growth software (Jones et al., 2003)

Pas de stress hydrique avant l'ouverture de la première capsule (SH0), stress hydrique en cours de cycle de culture (SH1), dès la floraison en trait plein foncé (flo) et durant le stade de remplissage des fruits en trait pointillé (remp) - No water stress before the opening of the first capsule (SHO), water stress during crop cycle (SH1), from the flowering trait full dark (flo) and during the filling stage of the dotted line results (remp). 
Tableau 4. Correspondance entre environnement et vécu hydrique estimé des cotonniers - Correspondence between environment and estimated water status of cotton.

\begin{tabular}{llll}
\hline Levée & Pluviosité & \multicolumn{2}{c}{ Vécu hydrique } \\
\cline { 2 - 4 } & & AMEX & SRD \\
\hline L1 : 1 au 30 juin & A & SH0 & SH0 \\
& B & SH0 & SH0 \\
& C & SH0 & - \\
L2 : 1 au 15 juillet & D & - & SH0 \\
& A & SH0 & - \\
& B & SH0 & SH0 \\
& C & SH1 & SH1 \\
L3 : 16 au 25 juillet & D & SH1 & SH1 \\
& B & - & SH1 \\
& C & SH1 & SH1 \\
L4 : après le 25 juillet & D & SH1 & SH1 \\
\hline
\end{tabular}

SH0 : pas de stress hydrique en cours de culture - no water stress during cultivation; $\mathrm{SH} 1$ : stress hydrique en cours de cycle - water stress during cultivation; A : installation des pluies précoce et pluviosité annuelle $>1000 \mathrm{~mm}$ - early arrival of rains (early May), annual rainfall $>1,000 \mathrm{~mm}$ and steady ; B : installation retardée et pluviosité annuelle $>1000 \mathrm{~mm}$ - late arrival of rains (late May-early June) and annual rainfall $>1,000 \mathrm{~mm} ; \mathrm{C}$ : installation retardée et pluviosité annuelle comprise entre 800 et $1000 \mathrm{~mm}$ - late arrival of rains and annual rainfall between 800 and 1,000 mm ; $\mathrm{D}$ : installation retardée et pluviosité annuelle $<800 \mathrm{~mm}-$ late arrival of rains and annual rainfall $<800 \mathrm{~mm}$.

vécu hydrique simulé des cotonniers, et c'est cette correspondance que nous avons adoptée comme règle pour estimer le vécu hydrique des cas non simulés à partir de leur environnement hydrique (95 sur 101). Pour les autres cas (6 sur 101), nous admettons qu'une levée précoce (L1) permet un cycle de culture sans stress hydrique, quels que soient le type de pluviosité et les conditions expérimentales d'AMEX ou de SRD. Sur l'ensemble des situations explorées, l'occurrence d'un stress hydrique (SH1) correspond respectivement à $9 \%, 27 \%$ et $47 \%$ des cas pour les zones agroclimatiques humide, intermédiaire et sèche.

Analyse statistique. Comme le montrent les tableaux 1 et $\mathbf{2}$, les variétés n'étant pas présentes sur l'ensemble des sites et des campagnes d'expérimentation, le dispositif à analyser est incomplet. Pour effectuer l'analyse, nous avons utilisé le logiciel SAS/STAT 9.12 (SAS Institute Inc., Cary, NC, USA) et plus précisément la procédure MIXED. Afin de prendre en compte le vécu hydrique dans le modèle linéaire adopté, nous avons considéré l'effet de la campagne agricole comme la somme d'un effet fixe du vécu hydrique des plantes et d'un effet aléatoire de l'année. Compte tenu des différences entre AMEX et SRD au niveau du dispositif et des pratiques agricoles, nous avons opté pour une analyse séparée des deux conditions expérimentales. Concernant la densité, des valeurs inférieures à 5 plantes $\mathrm{m}^{-2}$ ne permettent pas d'atteindre le potentiel de rendement escompté en condition d'alimentation hydrique non limitante (Dong et al., 2012). De plus, Sekloka et al. (2007) rapportent une modification sensible de la phénologie des plantes en fonction de la densité des plants. L'important effectif de densités inférieures à 5 plantes $\cdot \mathrm{m}^{-2}$ en condition de SRD contraint donc à introduire cette variable dans l'analyse, avec les deux modalités de $: \leq 5$ plantes $\cdot \mathrm{m}^{-2}$ (inf5) et $>5$ plantes $\cdot \mathrm{m}^{-2}$ ( $\sup 5$ ).

Les modèles statistiques adoptés sont les suivants :

$$
\begin{aligned}
\mathrm{Y}_{\mathrm{AMEX}}= & \operatorname{Var}+\mathrm{VH}+\mathrm{Site}+\mathrm{Bloc}(\text { Site })+(\operatorname{Var} \mathrm{x} \mathrm{VH})+ \\
& (\text { Var x Site })+\mathrm{An}+\mathrm{SI}+\varepsilon
\end{aligned}
$$

et

$$
\begin{aligned}
\mathrm{Y}_{\mathrm{SRD}}= & \operatorname{Var}+\mathrm{VH}+\text { Site }+ \text { Densité }+ \text { Paysan }(\text { Site })+ \\
& (\text { Var } x \mathrm{VH})+(\text { Var } x \text { Densité })+\mathrm{An}+\mathrm{SI}+\varepsilon
\end{aligned}
$$

avec Y : variable estimée, Var : effet fixe de la variété, VH : effet fixe du vécu hydrique, Densité : effet fixe de la densité de plants. $\mathrm{m}^{-2}$, Site : effet fixe du site expérimental, Bloc(site) : effet fixe du bloc en condition d'AMEX; Paysan(site) : effet fixe du paysan en condition de SRD; An : effet aléatoire de l'année d'essai; SI : somme des interactions (An $x$ Site), (An x Var) et $(\mathrm{An} \times \mathrm{VH}) ; \varepsilon$ : erreur.

Compte tenu de la nature incomplète du dispositif et de l'existence de quelques données manquantes, le logiciel SAS n'a pas toujours permis d'estimer la valeur des variables, d'où les manques dans les tableaux de résultats 5 à 7.

\section{RÉSULTATS}

\subsection{Caractéristiques agronomiques}

Conditions d'AMEX. Le tableau 5 présente les résultats de l'analyse statistique effectuée sur les variables de phénologie, le rendement en coton-graine, le rendement à l'égrenage et le rendement fibre. Sur les moyennes ajustées de l'effet du vécu hydrique, des différences significatives entre variétés s'observent sur la phénologie, FK 59K se démarquant des autres par sa précocité au moment de l'apparition de la première fleur et la date d'ouverture de la première capsule. Cette précocité ne procure toutefois pas d'avantage vis-à-vis des rendements en coton-graine et en fibre, pour lesquels aucune différence n'est observée entre variétés. Bien qu'un effet très significatif du vécu hydrique et du site soit observé, il n'y a pas d'interaction significative avec le génotype. Le rendement fibre n'a 
Tableau 5. Analyse statistique des tests réalisés en condition d'AMEX de 2004 à 2011 - Statistical analysis of tests in AMEX condition from 2004 to 2011.

\begin{tabular}{|c|c|c|c|c|c|c|c|}
\hline Variété & Vécu hydrique & O1F (jal) & FFU (jal) & O1C (jal) & Rdt CG $\left(\mathrm{kg} \cdot \mathrm{ha}^{-1}\right)$ & $\% \mathbf{F}(\%)$ & Rdt F $\left(\mathrm{kg} \cdot \mathrm{ha}^{-1}\right)$ \\
\hline \multicolumn{2}{|l|}{ Isco PG } & $58,2^{\mathrm{a}}$ & 76,2 & $103,1^{\mathrm{a}}$ & 1715 & $43,1^{b}$ & 824 \\
\hline \multicolumn{2}{|l|}{ Stam 279A } & $57,2^{\mathrm{ab}}$ & 76,0 & $101,8^{\mathrm{b}}$ & 1684 & $43,2^{\mathrm{b}}$ & 831 \\
\hline \multicolumn{2}{|c|}{ Stam 129A } & $57,1^{\mathrm{a} b}$ & 75,7 & $101,6^{\mathrm{b}}$ & 1612 & - & - \\
\hline \multicolumn{2}{|c|}{ Stam 59A } & $58,7^{\mathrm{a}}$ & 75,8 & $103,2^{\mathrm{ab}}$ & 1541 & $43,8^{b}$ & 866 \\
\hline \multicolumn{2}{|l|}{ FK $59 \mathrm{~K}$} & $56,2^{b}$ & 72,8 & $99,7^{\mathrm{c}}$ & 1464 & $45,1^{\mathrm{a}}$ & 884 \\
\hline & SHO & 58,3 & 77,9 & 103,3 & $1918^{a}$ & - & - \\
\hline & SH1 & 56,7 & 72,6 & 100,4 & $1289^{b}$ & - & - \\
\hline \multicolumn{8}{|c|}{ Niveau de significativité } \\
\hline \multicolumn{2}{|c|}{ Variété } & $*$ & ns & $* * *$ & ns & $* *$ & ns \\
\hline \multicolumn{2}{|c|}{ Vécu hydrique } & ns & ns & ns & $* *$ & - & - \\
\hline \multicolumn{2}{|c|}{ Site } & ns & ns & $*$ & $* *$ & ns & ns \\
\hline \multicolumn{2}{|c|}{ Variété x Vécu hydrique } & ns & ns & ns & ns & - & - \\
\hline \multicolumn{2}{|c|}{ Variété x Site } & ns & ns & ns & ns & ns & ns \\
\hline
\end{tabular}

$\mathrm{O} 1 \mathrm{~F}$ : date d'apparition de la première fleur - onset of the first flower ; FFU : date de fin de la floraison utile - end of flowering ; O1C : date d'ouverture de la première capsule - first boll opening; Rdt CG : rendement coton-graine - seed cotton yield ; \% F : rendement à l'égrenage - ginning outturn; Rdt F : rendement fibre - fiber yield ; SH0 : pas de stress hydrique en cours de culture - no water stress during cultivation; SH1 : stress hydrique en cours de cycle - water stress during cultivation; les variétés ou les vécus hydriques ne portant pas de lettre en commun sont statistiquement différents d'après le Test-t de Student à $5 \%$ - the cultivars or water status groups that do not share the same letter are significantly different from each other using Student t-Test at the 5\% level.

pas pu être estimé en conditions de stress hydrique des plantes (SH1). Un effet significatif de la variété est toutefois mis en évidence pour cette variable, avec un avantage remarquable de la variété FK 59K.

Conditions de SRD. Alors que des différences significatives entre les variétés s'observent en condition d'AMEX, aucune différence de phénologie ni de rendement n'est mise en évidence en condition de SRD (Tableau 6). On observe toutefois un effet du vécu hydrique sur le rendement en coton-graine et le rendement égrenage, tous deux affectés par un stress en cours de cycle. Sans effet sur le rendement en cotongraine, la densité de plantes $\cdot \mathrm{m}^{-2}$ a eu toutefois un effet significatif sur la date d'ouverture de la première capsule, avec un retard de 2 à 3 jours pour la plus haute densité. Enfin, l'analyse met en évidence l'importante variabilité de conditions de milieu d'un bloc à l'autre en parcelle d'agriculteur pour le rendement en fibre et résumé comme l'effet «paysan ».

\subsection{Caractéristiques commerciales}

Du fait de son absence dans les échantillons analysés, la variété témoin Stam 279A est aussi absente de l'analyse. Hormis pour la ténacité, un effet variétal est observé sur l'ensemble des autres caractéristiques (Tableau 7). Les seules caractéristiques affectées par le vécu hydrique des cotonniers sont l'Indice Micronaire (IM) et la finesse de la fibre $(\mathrm{H})$, avec toutefois une interaction non significative entre génotype et vécu hydrique. Les fibres de FK 59K sont plus longues, plus fines et plus blanches que celles de toutes les autres variétés. En termes de gain potentiel, la seule longueur des fibres de FK 59K permet de bénéficier d'un différentiel de prix de l'ordre de 1,25 US cent.pound ${ }^{-1}$ (soit environ 17 FCFA $\cdot \mathrm{kg}^{-1}$ de fibre) par rapport aux autres variétés, selon le National Cotton Council of America (2016). En outre, l'ensemble des caractéristiques de longueur, de micronaire, de ténacité et de couleur permettraient de bénéficier d'une prime de l'ordre de 4,75 US cents. pound $^{-1}$ (soit environ 64 FCFA. $\mathrm{kg}^{-1}$ de fibre). La «qualité » des fibres de FK 59K permettrait donc un gain potentiel de valeur financière d'environ $3 \%$ par rapport aux autres variétés. En ce qui concerne le couple finesse/maturité, les règles commerciales induisent des fortes décotes financières si le micronaire (IM) sort de la plage 3,5-4,9, ce qui n'est le cas d'aucune des variétés, même en condition de stress hydrique. Toute variété produisant des fibres fines court le risque de subir une décote financière alors même que leurs valeurs technologiques sont améliorées, davantage de fibres fines dans une section de fil donnée augmentant la résistance à la rupture des fils constitués. C'est le cas de la variété FK $59 \mathrm{~K}$, qui présente une faible masse linéique $(\mathrm{H})$. Toutefois, suite à des calculs spécifiques 
Tableau 6. Analyse statistique des tests réalisés en condition de SRD de 2004 à 2011 - Statistical analysis of tests in SRD condition from 2004 to 2011.

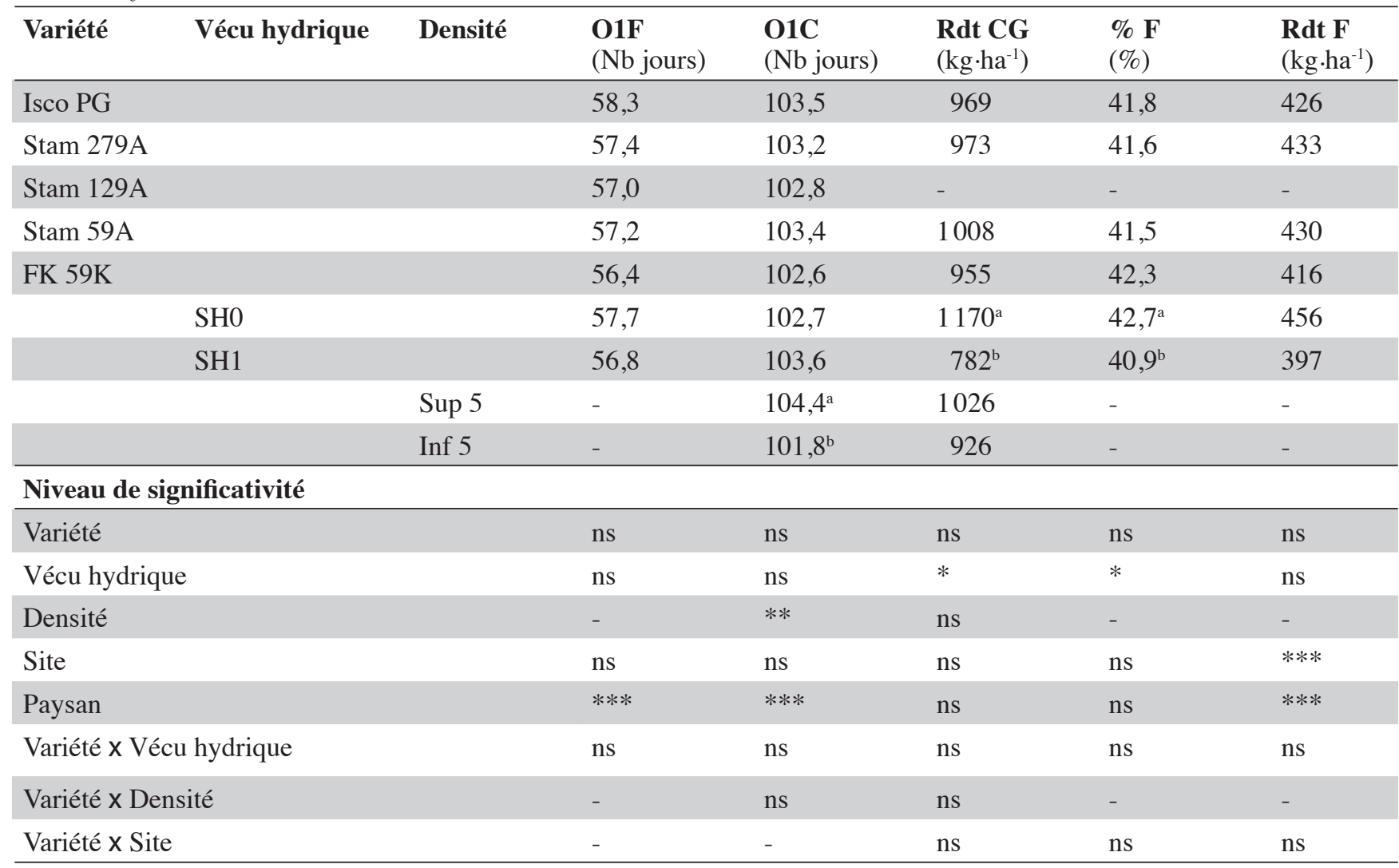

O1F, O1C, Rdt CG, \% F, Rdt F, SH0, SH1 : voir tableau 5 - see table 5 ; Sup 5 : densité supérieure ou égale à 5 plantes $\cdot \mathrm{m}^{-2}-$ density greater than or equal to 5 plants $\cdot m^{-2}$; Inf $5:$ densité inférieure à 5 plantes $\cdot \mathrm{m}^{-2}-$ density of less than 5 plants $\cdot m^{-2}$; les variétés ou les vécus hydriques ne portant pas de lettre en commun sont statistiquement différents d'après le Test-t de Student à $5 \%$ - the cultivars or water status groups that do not share the same letter are significantly different from each other using Student $t$-Test at the 5\% level.

Tableau 7. Analyse statistique des tests réalisés en condition d'AMEX en 2006 et 2009 - Statistical analysis of tests in AMEX condition in 2006 and 2009.

\begin{tabular}{|c|c|c|c|c|c|c|c|}
\hline Variété & Vécu hydrique & UHML $(\mathrm{mm})$ & $\mathbf{H}($ mtex $)$ & IM & Ténacité (CN tex $\left.{ }^{-1}\right)$ & $\mathbf{+ b}(\%)$ & $\mathbf{R d}(\%)$ \\
\hline Isco PG & & $27,9^{\mathrm{c}}$ & $164^{\mathrm{a}}$ & $4,2^{\mathrm{a}}$ & 28,7 & $8,7^{\mathrm{a}}$ & $75,8^{\mathrm{b}}$ \\
\hline Stam 129A & & $27,9^{\mathrm{bc}}$ & $159^{\mathrm{ab}}$ & $4,1^{\mathrm{a}}$ & 29,2 & $8,6^{\mathrm{a}}$ & $75,6^{b}$ \\
\hline Stam 59A & & $28,7^{\mathrm{ab}}$ & $153^{\mathrm{ab}}$ & $3,9^{\mathrm{b}}$ & 27,4 & $8,7^{\mathrm{a}}$ & $77,7^{\mathrm{a}}$ \\
\hline \multirow[t]{3}{*}{ FK 59K } & & $29,1^{\mathrm{a}}$ & $148^{\mathrm{b}}$ & $3,7^{\mathrm{b}}$ & 28,4 & $8,1^{\mathrm{b}}$ & $77,6^{\mathrm{a}}$ \\
\hline & SHO & 28,4 & $160^{\mathrm{a}}$ & $4,1^{\mathrm{a}}$ & 28,2 & 8,5 & 76,5 \\
\hline & SH1 & 28,3 & $152^{\mathrm{b}}$ & $3,8^{\mathrm{b}}$ & 28,6 & 8,5 & 76,9 \\
\hline \multicolumn{8}{|c|}{ Niveau de significativité } \\
\hline Variété & & $* *$ & $* * *$ & $* * *$ & $\mathrm{~ns}$ & $* * *$ & $* * *$ \\
\hline Vécu hydrique & & ns & $* *$ & $* *$ & ns & ns & ns \\
\hline Site & & $*$ & ns & $* *$ & $*$ & ns & ns \\
\hline Variété x Vécu & hydrique & ns & ns & ns & ns & ns & $*$ \\
\hline
\end{tabular}

UHML : longueur moyenne de la moitié supérieure - Upper High Mean Length; $\mathrm{H}$ : finesse linéique - linear fineness ; IM : Indice Micronaire - Micronaire Index ; +b : degré de jaune - degree of yellow ; Rd : la réflectance - reflectance ; SH0 : pas de stress hydrique en cours de culture - no water stress during cultivation; SH1 : stress hydrique en cours de cycle - water stress during cultivation; les variétés ou les vécus hydriques ne portant pas de lettre en commun sont statistiquement différents d'après le Test-t de Student à $5 \%$ - the cultivars or water status groups that do not share the same letter are significantly different from each other using Student $t$-Test at the $5 \%$ level. 
(non présentés), il semble que la maturité des fibres serait meilleure pour Stam 59A $(1,02)$ et Isco PG $(0,97)$, les deux autres variétés étant à 0,91 . Ces valeurs de maturité sont comprises dans l'intervalle $[0,9-1,0]$, soit la fourchette requise pour favoriser la filature et la prise de teinture. Pour la colorimétrie enfin $(\mathrm{Rd}$ et $+\mathrm{b})$, toutes les variétés sont groupées dans la même classe de « grade ». Cependant, en référence au classement du National Cotton Council of America (2016), Stam 59A bénéficierait d'une légère surprime par rapport à FK $59 \mathrm{~K}$, elle-même étant légèrement supérieure au groupe des variétés Isco PG et Stam 129A.

\section{DISCUSSION}

La méthodologie proposée dans l'article permet de caractériser le vécu hydrique des plantes et donc d'identifier l'occurrence d'un stress hydrique sévère durant les phases critiques de floraison et de développement des capsules vis-à-vis du rendement en coton-graine (de Kock et al., 1990). La distinction des deux catégories de vécu hydrique, $\mathrm{SH} 0$ et SH1, permet de discriminer les rendements en coton-graine dans les deux conditions d'AMEX et de SRD (Tableaux 5 et 6). Ce résultat constitue une forme de validation de la règle de correspondance adoptée pour accéder au vécu hydrique des plantes à partir de l'environnement hydrique tel qu'il a été défini sur la base des typologies de pluviosité et de plage de levée des cotonniers.

En condition expérimentale contrôlée d'AMEX et conformément à la littérature, un effet est observé sur les caractéristiques déterminées par le génotype, telle la phénologie (Sekloka et al., 2008), le rendement égrenage et la plupart des caractéristiques technologiques des fibres produites (Cao et al., 2011). La précocité de FK 59K, observée dès l'apparition de la première fleur, se retrouve en fin de cycle pour l'ouverture de la première capsule. Bien que significative, cette différence de précocité reste toutefois modeste avec un gain de 2 à 3 jours sur la durée de cycle par rapport aux témoins Isco PG et Stam 279A et n'apporte pas d'avantage en termes de productivité au champ dans les conditions environnementales explorées. En condition de SRD, par contre, et pour des niveaux de rendement en coton-graine moitié moindre qu'en condition d'AMEX, aucun effet variétal n'a été observé. Par ailleurs, et en cohérence avec les observations de Sekloka et al. (2015), un effet de la densité de plantes est observé sur la date d'ouverture de la première capsule en condition de SRD, avec un retard de quelques jours de la date d'ouverture des capsules pour les densités supérieures à 5 plantes $\cdot \mathrm{m}^{-2}$. Ce résultat va à l'encontre de la recommandation technique de la SODEFITEX d'une densité de 8 plantes $\cdot \mathrm{m}^{-2}$, bien supérieure à celle observée chez les producteurs animés par l'objectif d'évitement du stress hydrique en lien avec une date de semis tardive et/ou une fin de saison des pluies précoce. L'important écart de rendement en coton-graine entre les tests conduits en milieu réel et en milieu contrôlé, déjà rapporté par Lançon et al. (1989), est révélateur de conditions de culture sub-optimales en SRD par rapport à celles observées en AMEX. En termes d'interaction Génotype X Environnement, ce qui est donc testé en condition de SRD, c'est la rusticité des variétés vis-àvis des critères contrôlés (vécu hydrique et densité de plantes $\cdot \mathrm{m}^{-2}$ ) et des conditions de milieu spécifiques de chaque bloc en parcelle d'agriculteur (Table 6).

L'absence d'interaction entre variétés et vécu hydrique, quelles que soient les conditions de culture, optimales ou sub-optimales, met en évidence que les variétés ne diffèrent pas sur ce critère. Ce résultat est à rapprocher des observations de Clouvel et al. (2007) et Loison et al. (2017) qui rapportent un effet marqué de la sélection variétale sur le rendement égrenage et les caractéristiques commerciales des variétés à type indéterminé au Cameroun, mais sans augmentation du rendement moyen en coton-graine depuis 50 ans au niveau de la production nationale.

L'absence d'interaction observée entre vécu hydrique et variété est toutefois à relativiser. D'un côté, et du fait que les variétés relèvent du même type à croissance indéterminée et de la faible distance génétique entre elles, il est possible qu'elles ne diffèrent pas sur le plan de la résistance à la sècheresse et plus largement de la rusticité. De l'autre, il est possible que la distinction faite entre $\mathrm{SH} 0$ et $\mathrm{SH} 1$ ne permette pas de bien rendre compte de la complexité des interactions entre les variétés et leur environnement, tant abiotique que biotique. Ainsi, de Kock et al. (1990) observent un effet positif du stress sur les caractéristiques technologiques de la fibre lors de la maturation des capsules en fin de cycle au-delà de l'ouverture de la première capsule. Outre cet effet d'ordre physiologique, Renou \& Brévault. (2016) relatent l'effet intéressant d'un tel stress de fin de cycle vis-à-vis des insectes carpophages aux derniers stades larvaires et à un moindre titre les piqueurs-suceurs moins abondants sur les parcelles concernées.

Sur le plan méthodologique, le caractère incomplet du dispositif en condition d'AMEX (Tableau 1) nuit d'une part à l'analyse statistique, limitée par l'aptitude des modèles statistiques à estimer la valeur des variables, et d'autre part à l'évaluation du progrès génétique accompli depuis 2004, en référence notamment à la variété Stam 42, abandonnée dans les essais à partir de 2009. D'un point de vue technique, une façon de contourner la difficulté de conservation des témoins sur une longue période consiste à assurer des périodes de recouvrement entre témoins. Les outils statistiques sont alors capables de comparer des variétés sur une période longue, en référence à des témoins différant dans le temps (Bergonzini \& Duby, 1995). 
L'évaluation variétale par les utilisateurs finaux est essentielle, surtout dans les régions soumises à des stress hydriques où les interactions entre le génotype et le sol ainsi qu'avec le paysage sont les plus fortes (Fischer et al., 2012). Or, une importante variabilité intra-bloc s'observe chez de nombreux paysans (non montrée), vraisemblablement liée à l'hétérogénéité spatiale du sol à l'échelle du bloc expérimental. L'absence de répétition en parcelle d'agriculteur nuit donc à l'évaluation des variétés au niveau local (Piepho et al., 2011) et, par conséquent, à l'objectif principal des essais en condition de SRD.

Sur le plan de la valorisation de la production enfin, les variétés conduites en condition d'AMEX se distinguent sur les primes à la qualité générée par les caractéristiques de longueur, de finesse et de couleur des fibres. Sous réserve d'une stabilité de ces caractéristiques en conditions de culture réelle et sur les bases du rendement moyen en coton-fibre obtenu en condition de SRD et du cours de la fibre de 65 USD cents pound $^{-1}$ en 2016 (ICAC, 2016), les primes associées à la variété $\mathrm{FK} 59 \mathrm{~K}$ permettraient un gain économique potentiel pour la SODEFITEX de l'ordre de $8 \%$, soit 30000 FCFA $\cdot$ ha $^{-1}$.

\section{CONCLUSIONS}

En termes d'application, l'absence d'interaction entre les variétés et leur vécu hydrique suggère qu'une seule variété parmi celles testées pourrait être cultivée sur l'ensemble du bassin de production. Dans le cadre de cette option toutefois, la perte de rendement due à des conditions de vécu hydrique déficitaire est estimée à près de $600 \mathrm{~kg} \cdot \mathrm{ha}^{-1}$. En référence aux résultats de Cao et al. (2011) et Sekloka et al. (2007), des variétés à cycle plus court pourraient être testées pour pallier au risque climatique, principalement sur les zones sèche et intermédiaire. Par ailleurs, un gain économique pourrait être obtenu au niveau de la filière avec la variété FK 59K, en tablant sur une augmentation de la valeur marchande des fibres, à confirmer en condition de production réelle.

En référence aux modèles d'interaction entre génotype et environnement avancés par Desclaux et al. (2008), le schéma de sélection adopté par la SODEFITEX reste inscrit dans un mode productiviste reposant sur l'hypothèse d'un environnement standardisé du fait de l'application des recommandations techniques de la société cotonnière. Or, l'écart de performances observé entre les conditions d'AMEX et de SRD montre que ce n'est pas le cas. À dessein ou non selon l'environnement socio-économique, les pratiques des agriculteurs divergent des recommandations techniques, à l'exemple de la densité de plantes $\cdot$ ha $^{-1}$ ou de la date de levée dans ce qui a été mesuré en condition de SRD.

Afin d'améliorer l'efficience de la sélection de variétés rustiques adaptées au changement climatique et aux conditions de culture en milieu réel, il conviendrait d'une part de tendre à des dispositifs expérimentaux en AMEX plus représentatifs des conditions de culture rencontrées en milieu réel, en élargissant la gamme de date de semis, par exemple. D'autre part, en condition de SRD, il conviendrait d'améliorer l'évaluation des variétés par les utilisateurs finaux avec l'introduction de répétitions des tests au niveau des parcelles d'agriculteurs, par exemple.

Sur le plan méthodologique enfin, la méthodologie proposée dans l'article permet à l'expérimentateur d'accéder au vécu hydrique des plantes et donc à l'occurrence de stress hydrique à partir des données locales de pluviosité et de date de levée. Cette méthode s'applique aux conditions climatiques explorées de 2004 à 2011 et reste donc contingente de la variabilité des conditions climatiques explorées durant cette période. Dans l'hypothèse d'une variabilité accrue liée aux évolutions du changement climatique et dans l'objectif de la recherche de variétés adaptées, il conviendrait alors de renouveler les approches expérimentales en matière de métrologie. La mise en œuvre d'une sélection variétale appuyée par des critères physiologiques en est une illustration, à partir de l'assimilation photosynthétique maximale par exemple, que Ullah et al. (2008) ont montré fortement corrélée au rendement en coton-graine en conditions de stress hydrique.

\section{Bibliographie}

Badiane D., Gueye M.T., Coly E.V. \& Faye O., 2015. Gestion intégrée des principaux ravageurs du cotonnier au Sénégal et en Afrique occidentale. Int. J. Biol. Chem. Sci., 9(5), 2654.

Bergonzini J.-C. \& Duby C., 1995. Analyse et planification des expériences: les dispositifs en blocs. Issy-lesMoulineaux, France : Elsevier Mason SAS.

Cao T.-V. et al., 2011. Short-season cotton (Gossypium hirsutum) may be a suitable response to late planting in sub-Saharan regions. Field Crops Res., 120(1), 9-20.

Clouvel P., Michel-Dounias I., Pichot J.P. \& Crétenet M., 2007. Organisation de la production cotonnière africaine : de la décolonisation à la libéralisation des filières. In : Paul R., Aeschlimann J.-P. \& Feller Ch., eds. Histoire et agronomie : entre ruptures et durée. Paris : IRD, 229-246.

CORAF/WECARD, 2005. Catalogue des variétés de cotonnier sélectionnées. Année 2005, http://www. cottoninc.com/fiber/quality/Classification-Of-Cotton/, $(03 / 02 / 2017)$. 
Dacosta H., Kandia K.Y. \& Malou R., 2002. La variabilité spatio-temporelle des précipitations au Sénégal depuis un siècle. In : Actes du Colloque, Fourth International FRIEND Conference, March 2002, CapeTown, South Africa, 499-506.

de Kock J., de Bruyn L.P. \& Human J.J., 1990. The relative sensitivity to plant water stress during the reproductive phase of upland cotton (Gossypium hirsutum L.). Irrig. Sci., 11(4), 239-244.

Desclaux D. et al., 2008. Changes in the concept of genotype $\times$ environment interactions to fit agriculture diversification and decentralized participatory plant breeding: pluridisciplinary point of view. Euphytica, 163(3), 533-546.

Dong H., Li W., Eneji A.E. \& Zhang D., 2012. Nitrogen rate and plant density effects on yield and late-season leaf senescence of cotton raised on a saline field. Field Crops Res., 126, 137-144.

Fischer K.S. et al., 2012. Field phenotyping strategies and breeding for adaptation of rice to drought. Front. Physiol., 3, 282.

Hulme M., 1992. Rainfall changes in Africa: 1931-1960 to 1961-1990. Int. J. Climatol., 12(7), 685-699.

ICAC, 2016. Production and trade policies affecting the cotton industry - 2016, http://www.icac.org/cotton_info/ publications/statistics/stats_wtd/gm-e_2016.pdf, (03/02/2017).

Jones J.W. et al., 2003. The DSSAT cropping system model. Eur. J. Agron., 18(3-4), 235-265.

Lançon J., Chanselme J.L. ～\& Klassou C., 1989. Représentativité des essais variétaux conduits en milieu contrôlé, semi-réel ou réel dans la zone cotonnière du Nord-Cameroun. Coton Fibres Trop., 44(2), 117-126.

Loison R., 2015. Ecophysiological analysis and modeling of genotype by environment by crop management interactions on cotton (Gossypium hirsutum L.) in Cameroon for the design of ideotypes. $\mathrm{PhD}$ thesis: Université de Montpellier (France).

Loison R. et al., 2017. Sixty years of breeding in Cameroon improved fibre but not seed cotton yield. Exp. Agric., 53(2), 202-209.

Lopes C. et al., 2004. Transpiration of grapevines and cohabitating cover crop and weed species in a vineyard. A "snapshot" at diurnal trends. Vitis, 43(2), 111-117.

NASA, 2016. Prediction of worldwide energy resource (POWER) climatology resource for agroclimatology, https://power.larc.nasa.gov/cgi-bin/agro.cgi?na, (16/11/2016).

National Cotton Council of America, 2016. 2016 CCC Loan schedule of premiums and discounts for upland and ELS cotton, http://www.cotton.org/econ/govprograms/ cccloan/, (03/02/2017).

Ndong J.-B., 1995. L'évolution de la pluviométrie au Sénégal et les incidences de la sécheresse récente sur l'environnement. Rev. Géogr. Lyon, 70(3), 193-198.
New M. et al., 2006. Evidence of trends in daily climate extremes over southern and west Africa. J. Geophys. Res., 111(D14), D14102.

Nicholson S., 2005. On the question of the "recovery" of the rains in the West African Sahel. J. Arid Environ., 63(3), 615-641.

Oosterhuis D.M., Bourland F.M. \& Tugwell N.P., 1993. Physiological basis for the nodes-above-white-flower cotton monitoring system. In: Proceedings of the Beltwide Cotton Conference, National Cotton Council of America, Memphis, USA, 1181-1183.

Piepho H.-P. et al., 2011. Statistical aspects of on-farm experimentation. Crop Pasture Sci., 62(9), 721.

Renou A. \& Brévault T., 2016. Ravageurs et maladies du cotonnier, et gestion intégrée des ravageurs. In: Crétenet M. \& Gourlot J.-P., eds. Le cotonnier. Versailles, France : Quæ; CTA ; Gembloux, Belgique : Les Presses agronomiques de Gembloux, 109-154.

Sadras V.O. \& Milroy S.P., 1996. Soil-water thresholds for the responses of leaf expansion and gas exchange: a review. Field Crops Res., 47(2-3), 253-266.

Sagna P. et al., 2015. Les variations récentes du climat constatées au Sénégal sont-elles en phase avec les descriptions données par les scénarios du GIEC ? Pollut. Atmos., 227, 1-17.

Salack S. et al., 2012. Analyses multi-échelles des pauses pluviométriques au Niger et au Sénégal. Sécheresse, 23(1), 3-13.

Sekloka E. et al., 2007. Effective flowering time variations in upland cotton (Gossypium hirsutum) at different planting dates and stand densities in Benin. Exp. Agric., $\mathbf{4 3}(2), 173$.

Sekloka E. et al., 2008. Breeding new cotton varieties to fit the diversity of cropping conditions in Africa: effect of plant architecture, earliness and effective flowering time on late-planted cotton productivity. Exp. Agric., 44(2), 197-207.

Sekloka E., Lançon J., Batamoussi M. \& Thomas G., 2015. La réduction de la croissance végétative à forte densité de semis comme stratégie d'adaptation variétale aux semis tardifs en culture cotonnière pluviale au Bénin. Tropicultura, 33(4), 299-308.

Soumaré M. et al., 2008. Prévision de l'aire de diffusion des sorghos photopériodiques en Afrique de l'Ouest. Cah. Agric., 17(2), 160-164.

Sylla M.B., Diallo I.\& Sal J.S., 2013. West African monsoon in state-of-the-science regional climate models. In: Climate Variability - Regional and Thematic Patterns. Rijeka, Croatia: InTech.

Ullah I., Mehboob-ur-Rahman, Ashraf M. \& Zafar Y., 2008. Genotypic variation for drought tolerance in cotton (Gossypium hirsutum L.): leaf gas exchange and productivity. Flora Morphol. Distrib. Funct. Ecol. Plants, 203(2), 105-115.

(34 réf.) 